\title{
On LMI-based sliding mode control for uncertain discrete-time systems
}

\author{
Ahmadreza Argha, Li Li, Steven W. Su*, Hung Nguyen \\ Faculty of Engineering and Information Technology, University of Technology, Sydney, PO Box \\ 123, Broadway, NSW 2007, Australia
}

\begin{abstract}
In this paper, a new approach to design a robust discrete-time sliding mode control (DSMC) is proposed for uncertain discrete-time systems. To this end, an LMI approach is used to develop a new framework to design the sliding function which is linear to the state. Our proposed robust DSMC can be applied to unstable systems, and also there is no need to stabilize the underlying system first. It has been argued in the literature that for the systems involving balanced external disturbances, using switching component is not needed. In this paper, it is shown that with the assumption of smoothness of the external disturbances, a different form of switching element in the controller can outperform the so-called linear controller in terms of the thickness of the boundary layer around the sliding function and the ultimate bound on the system state. Also, this paper extends the idea of disturbance estimation to the uncertain discrete-time systems. The disturbance estimator is exploited in the controller design and the boundedness of the obtained closed-loop system is analyzed. Also, two novel forms of variable structure DSMC are suggested in this paper.
\end{abstract}

\footnotetext{
*Corresponding author

Email address: Steven. Su@uts. edu . au (Steven W. Su )
} 
Keywords: Uncertain discrete-time linear systems, discrete-time sliding mode control (DSMC), linear matrix inequalities (LMIs), disturbance estimator.

\section{Introduction}

In the continuous-time sliding mode control, to achieve ideal sliding mode, in general the control signal must switch at infinite frequency [1]. However, since in digital control strategies, the control signal is held constant during the sampling period, it is normally not possible to achieve ideal sliding. Hence, in uncertain discrete-time systems it is not possible to ensure that the system state remains certainly on a surface within the state space and consequently the DSMC problem is fundamentally different to its continuous-time counterpart [1]. In terms of DSMC, state trajectories would move within a vicinity of the predetermined sliding surface referred to as quasi-sliding mode band [2].

Although the early works on the DSMC aimed at establishing a discrete-time counterpart to the continuous-time reachability condition [2, 3, 4], it has been shown that DSMC does not necessarily require the use of a variable structure discontinuous control strategy (VSDCS) [5, 6, 7]. References [5, 6] have shown that the DSMC without VSDCS can ensure that the state trajectories stay within a neighbourhood of the sliding surface in the presence of bounded matched uncertainty. The obtained control law is called linear control law. Moreover, according to the results presented in $[5,6]$, the use of a switching function in the control law may not necessarily improve the performance. Note that, obviously, the DSMC problem using only linear control law can be regarded as a robust optimal control problem and it will be equivalent to discrete-time Lyapunov min-max problems [8] or discrete-time Riccati min-max problems [9]. Nevertheless, some papers in 
the literature have claimed a better performance thanks to the use of discontinuous components [7]. Indeed, these papers assume that either the sampling rate of the system is very high compared with the maximum frequency component of the exogenous disturbance or the exogenous disturbance is slow (smooth and bounded). With either of these assumptions, the closed-loop system would behave more or less as a continuous-time system [7] and hence, using a discontinuous component in the controller may improve the performance. In this paper, two new form of switching function is proposed which can be more efficient in terms of reducing the ultimate bound on the system state and reducing the chattering created by traditional switching functions. This new switching function, basically, uses a disturbance estimator which comes from the same idea presented in [10]. The idea of using disturbance observer for the DSMC was firstly presented in [10] and followed by e.g. $[11,12]$. The main idea is, with the assumption of continuity of the original continuous-time disturbance signal, to use the previous value of the sampled disturbance for estimating the current one in the control law. However, model uncertainty is not considered in [10]. In this paper, it is also discussed that using the mentioned estimator directly in the controller will increase the order of the system and, in addition, it results in a system involving time-delay. Stability analysis and ultimate boundedness is then investigated for this kind of systems.

It is worth mentioning that a novel implicit Euler numerical scheme has recently been proposed in $[13,14]$ that can avoid numerical chattering, by not using explicit (forward) methods of discretization. However, chattering appears again in the presence of disturbances. The basic idea is to implement the discontinuous input of the DSMC in an implicit form, while keeping its causality (i.e. the controller is non-anticipative). Then this input has to be computed at each sampling 
time as the solution to a generalized, set-valued equation, which takes the form of a simple projection on an interval in the simplest cases.

Also, note that the problem of designing the DSMC is mainly considered for the systems with matched uncertainty and/or external disturbance [1]. This paper greatly reduces the conservatism of the current LMI-based methods presented in the few existing works that consider the problem of applying DSMC to the systems including unmatched uncertainties. Specifically, this note avoids using inequalities to deal with the uncertain negative sign quadratic terms appeared in the derived Riccati-like inequality, which is not easy to be directly arranged as an LMI problem. Instead, a lossless technique is proposed to convert the mentioned inequality to a form that can be easily written as an LMI. This technique can extremely widen the feasible region of the derived LMI condition obtained for the design of robust sliding surface, and hence, the applicability region of our DSMC compared to the existing literature for the DSMC, e.g. see $[15,16]$. In brief, the proposed DSMC is a unified framework for general discrete-time LTI systems. This is significantly different from methods whose application is limited to the stable systems, $c f$. [15], and also the methods which need to pre-stabilize the system, $c f .[17]$.

The rest of this paper is organized as follows: Section 2 describes the problem formulation. In Section 3, the proposed method to design the sliding surface is given. Section 4 explains a more practical DSMC for the systems including uncertainty and disturbance. Different forms of DSMC are considered in Section 5. Efficiency of the proposed DSMC is studied by numerical examples in Section 6. Finally, Section 7 concludes this paper. 


\section{Problem Formulation}

Consider the following uncertain linear discrete-time system,

$$
x(k+1)=[A+\Delta A(k)] x(k)+B[u(k)+f(k)],
$$

where $x(k) \in \mathbb{R}^{n}$ and $u(k) \in \mathbb{R}^{m}$. Without loss of generality, it is assumed that $B \in \mathbb{R}^{n \times m}$ and $m \leq n$. Besides, $\operatorname{rank}(B)=m$ (matrix $B$ has full column rank) and it is assumed that the pair $(A, B)$ is stabilizable. The uncertain matrix $\Delta A(k)$ has the form of:

$$
\Delta A(k)=M R(k) N
$$

where matrices $M$ and $N$ are known and $R(k)$ is an unknown matrix satisfying $R^{T}(k) R(k) \leq I, \forall k \geq 0 ; f(k)$ denotes external disturbance with known bound, $\|f(k)\| \leq \bar{f}$, where $\bar{f}>0$. In the following of this paper, for simplicity, $\Delta A_{k}$ and $\Delta A_{k-1}$ will be used instead of $\Delta A(k)$ and $\Delta A(k-1)$, respectively.

The following materials are useful in the sequel.

Definition 1. Considering the uncertainty set $\mathscr{F}=\left\{F(k): F(k) F^{T}(k) \leq I, \forall k \geq\right.$ $0\}$, the matrix $\Theta$ is said to commute with the uncertainty $F(k) \in \mathscr{F}$ if it belongs to the commutant of the uncertainty set $\mathscr{F}$ as

$$
\Theta_{\mathscr{F}}=\{\Theta: \Theta \text { is invertible and } F(k) \Theta=\Theta F(k), \forall F(k) \in \mathscr{F}\} \text {. }
$$

Also, we define the positive commutant set

$$
\boldsymbol{P} \Theta_{\mathscr{F}}=\left\{\bar{\Theta}: \bar{\Theta}=\Theta \Theta^{T}>0, \forall \Theta \in \Theta_{\mathscr{F}}\right\} .
$$

Lemma 1. Let $E, F(k)$ and $H$ be real matrices of appropriate dimensions with $F(k) \in \mathscr{F}$, then, for any matrix $\bar{\Gamma} \in P \Theta_{\mathscr{F}}$, we have

$$
E F(k) H+H^{T} F^{T}(k) E^{T} \leq E \bar{\Gamma} E^{T}+H^{T} \bar{\Gamma}^{-1} H .
$$


Proof. Note that $\bar{\Gamma}=\Gamma \Gamma^{T}, \Gamma \in \boldsymbol{\Theta}_{\mathscr{F}}$. Then it can easily be proved by

$$
\left[E F(k) \Gamma-H^{T}\left(\Gamma^{T}\right)^{-1}\right]\left[\Gamma^{T} F^{T}(k) E^{T}-\Gamma^{-1} H\right] \geq 0 .
$$

Note that a similar lemma is given in [18] which is a specific form of the above Lemma with $\bar{\Gamma}=\varepsilon I$, where $\varepsilon>0$ is a scalar. In addition, Lemma 1 can be specified to the following particular form, which will be used in the sequel of this paper.

Corollary 1. Let $E=\left[\begin{array}{ll}E_{1} & E_{2}\end{array}\right], \Delta(k)=\left[\begin{array}{cc}F(k) & 0 \\ 0 & F(k-1)\end{array}\right]$ and $H=\left[\begin{array}{l}H_{1} \\ H_{2}\end{array}\right]$ be real matrices of appropriate dimensions with $F(k) \in \mathscr{F}$, then, for any scalars $\varepsilon_{i}>0$, $i=1,2$, we have

$$
E \Delta(k) H+H^{T} \Delta^{T}(k) E^{T} \leq E\left[\begin{array}{cc}
\varepsilon_{1} I & 0 \\
0 & \varepsilon_{2} I
\end{array}\right] E^{T}+H^{T}\left[\begin{array}{cc}
\varepsilon_{1}^{-1} I & 0 \\
0 & \varepsilon_{2}^{-1} I
\end{array}\right] H
$$

Lemma 2. Consider the following inequality:

$$
\Gamma\left(X_{1}, X_{2}, \cdots, X_{n}\right)-\sum_{i=1}^{n} \digamma_{i}^{T}\left(X_{i}\right) \Lambda_{i}^{-1}\left(X_{i}\right) \digamma_{i}\left(X_{i}\right)<0,
$$

where $X_{i}, i=1, \cdots, n$ are the matrix variables, $\Lambda_{i}\left(X_{i}\right)>0$ and $\digamma_{i}\left(X_{i}\right)$ are functions of $X_{i}, i=1, \cdots, n$. Then the inequality in (3) is feasible in $X_{i}, i=1, \cdots, n$ if and only if the following inequality is feasible in $X_{i}, J_{i}, i=1, \cdots, n$ :

$$
\Gamma\left(X_{1}, X_{2}, \cdots, X_{n}\right)+\sum_{i=1}^{n}\left(J_{i}^{T} \Lambda_{i}\left(X_{i}\right) J_{i}+J_{i}^{T} \digamma_{i}\left(X_{i}\right)+\digamma_{i}^{T}\left(X_{i}\right) J_{i}\right)<0 .
$$

PROOF. It can be shown that the feasibility in $X_{i}, i=1, \cdots, n$ of (3) is equivalent to the feasibility in $X_{i}, J_{i}, i=1, \cdots, n$ of

$$
\begin{aligned}
& \Gamma\left(X_{1}, X_{2}, \cdots, X_{n}\right)-\sum_{i=1}^{n} \digamma_{i}^{T}\left(X_{i}\right) \Lambda_{i}^{-1}\left(X_{i}\right) \digamma_{i}\left(X_{i}\right) \\
& +\sum_{i=1}^{n}\left(\left[J_{i}+\Lambda_{i}^{-1}\left(X_{i}\right) \digamma_{i}\left(X_{i}\right)\right]^{T} \Lambda_{i}\left(X_{i}\right)\left[J_{i}+\Lambda_{i}^{-1}\left(X_{i}\right) \digamma_{i}\left(X_{i}\right)\right]\right)<0,
\end{aligned}
$$


where $J_{i}, i=1, \cdots, n$ are introduced auxiliary variables [19]. Indeed, the inference from (5) to (3) is obvious, and the inference from (3) to (5) follows by letting $J_{i}=-\Lambda_{i}^{-1}\left(X_{i}\right) \digamma_{i}\left(X_{i}\right)$. Then, it is easy to show that (5) is equivalent to (4). This completes the proof.

\section{Design of the Discrete-time SMC}

Consider the following linear discrete-time sliding function:

$$
\sigma_{x}(k)=S x(k)
$$

where $S \in \mathbb{R}^{m \times n}$ will be designed later such that $S B$ is nonsingular. During the ideal sliding motion the sliding function satisfies:

$$
\sigma_{x}(k+1)=\sigma_{x}(k)=0, \forall k>k_{s}
$$

where $k_{s}>0$ denotes the time that sliding motion starts. Thus, one may obtain from (1) and (6) that

$$
\sigma_{x}(k+1)=S\left(A+\Delta A_{k}\right) x(k)+S B[u(k)+f(k)]
$$

Here we will provide the mean value and boundary layer thickness vectors for the exogenous disturbance according to the upper and lower bounds of $f(k)$. In doing so, assume

$$
f_{i}^{l} \leq f_{i}(k) \leq f_{i}^{u}, \quad i=1, \cdots, m
$$

where $f_{i}^{l}$ and $f_{i}^{u}$ denote the lower and upper bound of the $i$-th entry of $f(k)$. Define

$$
f_{i}^{+}=\frac{f_{i}^{u}+f_{i}^{l}}{2}, f_{i}^{-}=\frac{f_{i}^{u}-f_{i}^{l}}{2}, i=1, \cdots, m
$$

and

$$
\mathscr{F}^{+}=\operatorname{col}\left(f_{1}^{+}, \cdots, f_{m}^{+}\right), \mathscr{F}^{-}=\operatorname{col}\left(f_{1}^{-}, \cdots, f_{m}^{-}\right)
$$


where $\mathscr{F}^{+}$and $\mathscr{F}^{-}$are the mean value and boundary layer thickness vectors of $f(k)$ respectively. Now, the following control law is proposed:

$$
u(k)=-(S B)^{-1} S A x(k)-\vartheta(k)
$$

where $\vartheta(k)$ denotes the approximation of disturbance $f(k)$ which may be used in the controller to compensate the bad effect of disturbance on the ultimate bound on the system state trajectories. $\vartheta(k)$ can also be regarded as the feedforward control, in addition to the linear controller. It is assumed that the component $\vartheta(k)$ is bounded, satisfying

$$
\|f(k)-\vartheta(k)\| \leq \tau\left\|\mathscr{F}^{-}\right\|
$$

where $\tau$ is a predefined positive scalar depending on the choice of $\vartheta(k)$. More discussions about the component $\vartheta(k)$ and $\tau$ are presented later in this paper.

Remark 1. In this note, the control law (12) uses only the information of the upper and lower bounds on the matched exogenous disturbance. It can be seen in the literature $([15,20,21])$ that the term $(S B)^{-1} S \Delta A x(k)$ is usually assumed to be bounded and its bound is exploited in the nonlinear part of SMC to ensure the reachability of the designed switching function. Nevertheless, the reason that we would not use the bound on this term is twofold. Firstly, as stated in e.g. [5, 6, 7], a linear control law in discrete-time can enure a bounded motion around the sliding surface in the presence of bounded matched uncertainty. Furthermore, it will be assumed in this paper that with a proper choice of $S$ and due to small unmatched uncertainty $\Delta A$, the magnitude of the term $(S B)^{-1} S \Delta A x(k)$ is negligible compared to the exogenous disturbance. 


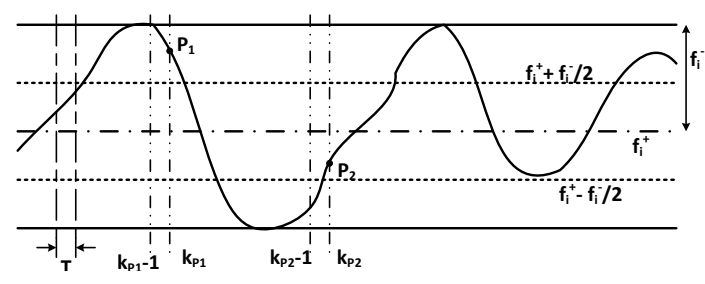

Figure 1: Signal $f_{i}(k)$

\subsection{Variable structure discontinuous control considerations}

As discussed in section 2, in the literature, it is argued that the discontinuous part of the sliding control input can be detrimental to performance [1]. However, this claim is only true for the balanced uncertainties and/or disturbances whose maximum frequency component is close to the sampling rate of the discrete-time system. Specifically, with the smoothness and boundedness conditions of the external disturbance, a number of beneficial choices as discontinuous variable structure components can be utilized in the DSMC in order to improve its performance. To explain, assume that $f_{i}(k)$ (the $i$ th element in $\left.f(k)\right)$ has the waveform as in Figure 1. Now, for instance, to estimate the instantaneous amplitude of disturbance at point $P_{1}$, five choices are accessible: 1) zero, 2) $f_{i}^{+}$, 3) $\left.f_{i}^{+}+f_{i}^{-}, 4\right) f_{i}^{+}+\frac{f_{i}^{-}}{2}$, 5) $f_{i}\left(k_{P_{1}}-1\right)$. Similarly, for point $P_{2}$, one may suggest to use 1) zero, 2) $f_{i}^{+}, 3$ ) $f_{i}^{+}-f_{i}^{-}$, 4) $\left.f_{i}^{+}-\frac{f_{i}^{-}}{2}, 5\right) f_{i}\left(k_{P_{2}}-1\right)$. Here, $f_{i}\left(k_{P_{j}}-1\right)$ means the value of $f_{i}$ at the time instant of $k=k_{P_{j}}-1, j=1,2$. Using the first choice (or indeed the lack of any discontinuous component) in the controller leads to the well-known linear controller. Exploiting the second choice, referred to as the mean value of the exogenous disturbance, in the DSMC has been proposed in [5]. It is presented in [5] that the term $f_{i}^{+}$can be used in the $i$ th element of the control law to compensate the nonzero mean of unbalanced disturbances. It can easily be realized that in 
the case of using $f_{i}^{+}$the maximum estimation error is $f_{i}^{-}$. In the following of this subsection, according to the third and fourth choices, we will discuss two different form of VSDC for DSMC. The discussion about the last choice, which is referred to as disturbance observer, will be the subject of the next section. In what follows, we assume that the exogenous disturbance in system (1) is smooth and bounded.

Assumption 1. The exogenous disturbance $f(k)$ in (1) satisfies the Lipschitz continuity condition and we have,

$$
\|f(k)-f(k-1)\| \leq L_{f} T_{s}
$$

where $L_{f}>0$ denotes Lipschitz constant and $T_{s}$ is the sampling time.

Here, it will be assumed that $L_{f}$ has a small value. To this end, the sampling rate of the discrete signal processing system is assumed to be big enough compared to the maximum component frequency of exogenous disturbance $f(k)$. Further in what follows, we assume the known sliding surface matrix $S$ and its design will be derived in Section 3.2.

\subsubsection{Using upper and lower bounds of disturbance in the controller: $\mathscr{C}_{1}$}

Note that

$$
f(k-1)=(S B)^{-1} S\left[x(k)-A x(k-1)-\Delta A_{k-1} x(k-1)-B u(k-1)\right] .
$$

$f(k-1)$ may be estimated by:

$$
\hat{f}(k)=(S B)^{-1} S[x(k)-A x(k-1)-B u(k-1)],
$$

which is equivalent to

$$
\hat{f}(k)=(S B)^{-1} S \Delta A_{k-1} x(k-1)+f(k-1) .
$$


For zero-centered uncertainty $\Delta A$, it is obvious that the term $(S B)^{-1} S \Delta A_{k-1} x(k-$ $1)$ is also zero-centered and has no influence on the mean values of the vector $\hat{f}(k)$. Additionally, in the case that system state is bounded, the vector $(S B)^{-1} S \Delta A_{k-1} x(k-$ 1) remains also bounded. With the proper choice of $S$ and for small uncertainty $\Delta A$, it can be claimed that the magnitude of $(S B)^{-1} S \Delta A_{k-1} x(k-1)$ will be very small compared to $f(k-1)$. Traditionally, sign function can be used to determine the position of the instantaneous disturbance relative to the line $f_{i}^{+}$. Hence, one may propose to set $\vartheta(k)$ in (12) as:

$$
\vartheta_{1}(k)=\mathscr{F}^{+}+\operatorname{diag}\left(\mathscr{F}^{-}\right) \operatorname{sgn}\left(\hat{f}(k)-\mathscr{F}^{+}\right)
$$

where $\operatorname{diag}\left(\mathscr{F}^{-}\right):=\operatorname{diag}\left(f_{1}^{-}, \cdots, f_{m}^{-}\right)$. Thus, the controller (12) can be defined as:

$$
u_{1}(k)=-(S B)^{-1} S A x(k)-\mathscr{F}^{+}-\operatorname{diag}\left(\mathscr{F}^{-}\right) \operatorname{sgn}\left(\hat{f}(k)-\mathscr{F}^{+}\right) .
$$

Remark 2. With a quick glimpse into the literature, it can be found that a frequently used candidate for the component $\vartheta(k)$ has the general form of:

$$
\vartheta(k)=\psi+v \operatorname{sgn}\left(\sigma_{x}(k)\right)
$$

where $\psi$ and $v$ are known parameters. For instance, in [15], with ignoring the bounds of $S \Delta A_{k} x(k)$ (see Remark 1), $\psi$ and $v$ are assumed to be some constants involving the bounds of $\operatorname{SBf}(k)$, similar to $\mathscr{F}^{+}$and $\mathscr{F}^{-}$. Regardless of different approaches used to design the parameters of this nonlinear function, it should be emphasized that the term $\operatorname{sgn}\left(\sigma_{x}(k)\right)$ is not an appropriate function to determine the position of the disturbance relative to its mean value either in the physical meaning or in the theoretical sense. Using the controller containing $\vartheta(k)$ as in (19) will lead state trajectories to chatter around the switching surface with 
amplitude dependent on the lower bound of the term (19) and with the frequency equal to the sampling rate; see [5]. Using the controller (18), while the chattering still happens, in this case, the state trajectories chatter with the frequency equal to the frequency of exogenous disturbance.

\subsection{2. $U \operatorname{sing} f_{i}^{+} \pm \frac{f_{i}^{-}}{2}: \mathscr{C}_{2}$}

As a new alternative, $f_{i}^{+} \pm \frac{f_{i}^{-}}{2}$ can be used as an estimate of $P_{1}$ or $P_{2}$ in Figure 1. The estimation error, in the worst-case scenario, will be $\frac{3}{2} f_{i}^{-}$. Hence, one may propose to put the component $\vartheta(k)$ in (12) as:

$$
\vartheta_{2}(k)=\mathscr{F}^{+}+\frac{1}{2} \operatorname{diag}\left(\mathscr{F}^{-}\right) \operatorname{sgn}\left(\hat{f}(k)-\mathscr{F}^{+}\right) .
$$

Thus, the controller (12) is chosen as:

$$
u_{2}(k)=-(S B)^{-1} S A x(k)-\mathscr{F}^{+}-\frac{1}{2} \operatorname{diag}\left(\mathscr{F}^{-}\right) \operatorname{sgn}\left(\hat{f}(k)-\mathscr{F}^{+}\right),
$$

where $\hat{f}(k)$ is defined in (16).

\subsection{Design of the robust sliding surface}

The sequel of this section aims to consider the stability of the system (1) using the controller (12). As a result of applying the controller (12) to the system (1), it is seen that

$$
x(k+1)=\left(A+\Delta A_{k}-\hat{A}\right) x(k)+B f_{\vartheta}(k)
$$

where $f_{\vartheta}(k) \triangleq f(k)-\vartheta(k)$ and $\hat{A} \triangleq B(S B)^{-1} S A$. Furthermore, it can be found that

$$
\sigma_{x}(k+1)=S \Delta A_{k} x(k)+S B f_{\vartheta}(k)
$$

The following lemmas are given to characterize the boundedness of the system state (22). 
Lemma 3 ([22]). Let $V(\zeta(k))$ be a Lyapunov candidate function. In the case that there exist real scalars $v \geq 0, \alpha>0, \beta>0$ and $0<\rho<1$ such that

$$
\alpha\|\zeta(k)\|^{2} \leq V(\zeta(k)) \leq \beta\|\zeta(k)\|^{2},
$$

and

$$
V(\zeta(k+1))-V(\zeta(k)) \leq v-\rho V(\zeta(k)),
$$

then $\zeta(k)$ will satisfy

$$
\|\zeta(k)\|^{2} \leq \frac{\beta}{\alpha}\|\zeta(0)\|^{2}(1-\rho)^{k}+\frac{\nu}{\alpha \rho} .
$$

Lemma 4. For any symmetric matrix $P>0$ and any full column rank matrix $B$, we have $P B\left(B^{T} P B\right)^{-1} B^{T} P \leq P$.

PROOF. It can easily be proved by

$$
\left[I-B\left(B^{T} P B\right)^{-1} B^{T} P\right]^{T} P\left[I-B\left(B^{T} P B\right)^{-1} B^{T} P\right] \geq 0 .
$$

It should also be noted that with applying DSMC to discrete-time systems involving exogenous disturbances, the closed-loop system should be analyzed in terms of boundedness. Also, the DSMC can only ensure that the state trajectories may be driven into a boundary layer around the ideal sliding surface $\sigma(k)=0$. This issue is indeed regarded as the quasi sliding mode (QSM) in the literature. On the other hand, due to the presence of mismatched uncertainty in the system dynamics, it is difficult to analyze the reachability of the QSM by means of a separate sufficient condition. Alternatively, the following theorem considers a method to analyze simultaneously the reachabiltiy of QSM and the stability of the system states by means of a discrete-time Lyapunov stability method. 
Theorem 1. In the absence of disturbance $f(k)$, the linear part of the control law (12) can drive the system state onto the ideal sliding surface (6), and the system state is stabilized, if there exist a symmetric matrix $\bar{P}>0$, matrices $X$ and $Y$, and scalars $\varepsilon>0$ and $\bar{\eta}>0$ satisfying the following LMI:

$$
\left[\begin{array}{cccccc}
-\bar{P}+Y^{T} B^{T}+B Y & \star & \star & \star & \star & \star \\
0 & -\bar{P}+2 \varepsilon M M^{T} & \star & \star & \star & \star \\
A \bar{P}+B X & \sqrt{2} \varepsilon M M^{T} & -\bar{P}+\varepsilon M M^{T} & \star & \star & \star \\
B Y & 0 & 0 & -\bar{P} & \star & \star \\
\bar{P} & 0 & 0 & 0 & -\bar{\eta} I & \star \\
N \bar{P} & 0 & 0 & 0 & 0 & -\varepsilon I
\end{array}\right]<0,
$$

where $M$ and $N$ are known matrices in (2). Here $S=B^{T} \bar{P}^{-1}$ and $\{\star\}$ denotes the symmetric elements in a symmetric matrix.

ProOF. Define

$$
V(\zeta(k))=x^{T}(k) P x(k)+\sigma_{x}^{T}(k)(S B)^{-1} \sigma_{x}(k),
$$

where $P>0$ is a symmetric matrix and $S=B^{T} P$. Thus, we can write

$$
\begin{aligned}
\Delta V(\zeta(k))= & V(\zeta(k+1))-V(\zeta(k)) \\
= & x^{T}(k+1) \operatorname{Px}(k+1)+\sigma_{x}^{T}(k+1)(S B)^{-1} \sigma_{x}(k+1) \\
& -x^{T}(k) \operatorname{Px}(k)-\sigma_{x}^{T}(k)(S B)^{-1} \sigma_{x}(k) .
\end{aligned}
$$

Now, it can be shown that

$$
\Delta V(\zeta(k))=\left[\begin{array}{c}
x(k) \\
f_{\vartheta}(k)
\end{array}\right]^{T}\left[\begin{array}{ll}
\Omega_{11} & \Omega_{12} \\
\Omega_{12}^{T} & \Omega_{22}
\end{array}\right]\left[\begin{array}{c}
x(k) \\
f_{\vartheta}(k)
\end{array}\right],
$$


where

$$
\begin{aligned}
\Omega_{11}:= & \left(A+\Delta A_{k}\right)^{T} P\left(A+\Delta A_{k}\right)-\left(A+\Delta A_{k}\right)^{T} P B\left(B^{T} P B\right)^{-1} B^{T} P\left(A+\Delta A_{k}\right) \\
& -P-P B\left(B^{T} P B\right)^{-1} B^{T} P+2 \Delta A_{k}^{T} P B\left(B^{T} P B\right)^{-1} B^{T} P \Delta A_{k}, \\
\Omega_{12}:= & 2 \Delta A_{k}^{T} S^{T}, \\
\Omega_{22}:= & 2 S B,
\end{aligned}
$$

In the absence of the disturbance $f(k)$, that is $f(k)=0$, thus $\vartheta(k)=0$ leading to $f_{\vartheta}(k)=0$. Then the system is stabilized if

$$
\Omega_{11}<-\eta I
$$

where $\eta>0$ is a scalar variable. Now, we consider the feasibility of (28). To obtain (28), by utilizing Lemma 4 and the Schur complement, it suffices to have

$$
\left[\begin{array}{cc}
\hat{\Omega}_{11} & \sqrt{2} \Delta A_{k}^{T} \\
\sqrt{2} \Delta A_{k} & -\bar{P}
\end{array}\right]<0,
$$

where $\bar{P}=P^{-1}$, and

$$
\begin{aligned}
\hat{\Omega}_{11}= & \left(A+\Delta A_{k}\right)^{T} P\left(A+\Delta A_{k}\right)-\left(A+\Delta A_{k}\right)^{T} P B\left(B^{T} P B\right)^{-1} B^{T} P\left(A+\Delta A_{k}\right) \\
& -P-P B\left(B^{T} P B\right)^{-1} B^{T} P+\eta I .
\end{aligned}
$$

According to Lemma 2, the feasibility of the inequality in (29) is equivalent to that of

$$
\left[\begin{array}{cc}
\tilde{\Omega}_{11} & \sqrt{2} \Delta A_{k}^{T} \\
\sqrt{2} \Delta A_{k} & -\bar{P}
\end{array}\right]<0,
$$

where

$$
\begin{aligned}
\tilde{\Omega}_{11}= & \left(A+\Delta A_{k}+B F\right)^{T} P\left(A+\Delta A_{k}+B F\right)-P+\eta I \\
& +L^{T}\left(B^{T} P B\right) L+L^{T} B^{T} P+P B L .
\end{aligned}
$$


Here, $F$ and $L$ are two auxiliary variables [19]. Then, by left and right matrix multiplication on both sides of the inequality in (30) with $\operatorname{diag}(\bar{P}, I)$, we have

$$
\left[\begin{array}{cc}
\bar{P} \tilde{\Omega}_{11} \bar{P} & \sqrt{2} \bar{P} \Delta A_{k}^{T} \\
\sqrt{2} \Delta A_{k} \bar{P} & -\bar{P}
\end{array}\right]<0 .
$$

Using the Schur complement and Lemma 1, it can be demonstrated that the inequality in (31) can be implied by the LMI in (24), where $X=F \bar{P}, Y=L \bar{P}$ and $\bar{\eta}=\eta^{-1}$.

Remark 3. It is worth mentioning that as $\zeta=\left[\begin{array}{ll}x^{T} & \sigma^{T}\end{array}\right]^{T}=\left[\begin{array}{c}I_{B^{T} P}\end{array}\right] x$ and $\left[\begin{array}{c}{ }_{B^{T} P}\end{array}\right]$ is a full rank matrix, $\zeta=0$ if and only if $x=0$. In addition, a key feature in our method to prove Theorem 1 (and Theorem 3 in the following of the paper), and further design the sliding function matrix $S$, is to neglect the bounded inputs (e.g., the nonlinear control and exogenous disturbance), and directly prove the stability of the unforced linear system. More precisely, from (27) (with $\left.f_{\vartheta}(k)=0\right)$ and (28) we may write

$$
\begin{aligned}
\Delta V(\zeta) & :=x^{T}(k) \Omega_{11} x(k) \\
& \leq-\eta x^{T}(k) x(k) \\
& \leq-\frac{\eta}{\lambda_{\max }\left(P+P B\left(B^{T} P B\right)^{-1} B^{T} P\right)} x^{T}(k)\left\{P+P B\left(B^{T} P B\right)^{-1} B^{T} P\right\} x(k) \\
& \triangleq-\rho V(\zeta),
\end{aligned}
$$

which ensures the asymptotic stability of the closed-loop system and thus $\zeta \rightarrow 0$, $\sigma \rightarrow 0$ and $x \rightarrow 0$.

Remark 4. The proof of this theorem provides a less conservative sufficient condition for the design of a robust sliding matrix for the system in (1) involving mismatched uncertainties. Further based on this proof, the second objective of 
this paper, when the disturbance estimator is utilized in the controller directly, will be derived in the proof of Theorem 3.

\subsection{Characterizing the system state boundedness}

While Theorem 1 presents a method to design the DSMC in order to stabilize the system in (1), it does not present a bound on the system states. The following theorem characterizes the boundedness of the obtained closed-loop system state and corresponding sliding function.

Theorem 2. In the presence of disturbance $f(k)$, if the LMI in (24) is feasible, for the obtained $P=\bar{P}^{-1}$ and $\eta=\bar{\eta}^{-1}$, the controller (12) satisfying (13) will lead to a bound on the augmented system state $\zeta(k)=\left[x^{T}(k), \sigma_{x}^{T}(k)\right]^{T}$ as follows:

$$
\begin{aligned}
& \forall \varsigma>0, \exists k^{\star}>0, \text { s.t. } \forall k>k^{\star}, \\
& \|\zeta(k)\|^{2} \leq \frac{\lambda_{\max }(\mathbf{M})}{\hat{\eta} \lambda_{\min }\left(\operatorname{diag}\left(P,\left(B^{T} P B\right)^{-1}\right)\right)} \gamma+\varsigma,
\end{aligned}
$$

where $\mathbf{M}=P B\left(B^{T} P B\right)^{-1} B^{T} P+P$, and $\gamma=\tau^{2}\left\|\mho+2 B^{T} P B\right\|\left\|\mathscr{F}^{-}\right\|^{2}$; here the scalar variable $\hat{\eta}>0$ and matrix variable $\mho>0$ are obtained from solving the following LMI:

$$
\left[\begin{array}{ccc}
(\hat{\eta}-\eta) I+4 \bar{\varepsilon} N^{T} N & \star & \star \\
0 & -\mho & \star \\
0 & M^{T} P B & -\bar{\varepsilon} I
\end{array}\right]<0,
$$

where $M$ and $N$ are known matrices in (2), and further, $\bar{\varepsilon}>0$ is a scalar variable.

Proof. According to Lemma 1 (by assuming $F=I$ ) it can be written that

$$
2 x^{T}(k) \Omega_{12} f_{\vartheta}(k) \leq x^{T}(k) \Omega_{12} \mho^{-1} \Omega_{12}^{T} x(k)+f_{\vartheta}^{T}(k) \mho f_{\vartheta}(k),
$$


where $\mho>0$. It follows from (27), (28) and (34) that

$$
\begin{aligned}
\Delta V(\zeta(k)) \leq & -x^{T}(k)\left\{\eta I-\Omega_{12} \mho^{-1} \Omega_{12}^{T}\right\} x(k) \\
& +f_{\vartheta}^{T}(k)\left[\mho+\Omega_{22}\right] f_{\vartheta}(k) .
\end{aligned}
$$

If we choose $\mho>0$ such that

$$
\hat{\eta} I<\eta I-\Omega_{12} \mho^{-1} \Omega_{12}^{T}
$$

where $0<\hat{\eta}<\eta$, which is always possible if $\eta>0$ exists, then it follows from (35) that

$$
\Delta V(\zeta(k)) \leq-\hat{\eta} x^{T}(k) x(k)+f_{\vartheta}(k)^{T}\left[\mho+\Omega_{22}\right] f_{\vartheta}(k)
$$

Note also that

$$
\begin{aligned}
V(\zeta(k)) & =x^{T}(k)\left[P+P B\left(B^{T} P B\right)^{-1} B^{T} P\right] x(k) \\
& \triangleq x^{T}(k) \mathbf{M} x(k),
\end{aligned}
$$

hence,

$$
\lambda_{\min }(\mathbf{M})\|x(k)\|^{2} \leq V(\zeta(k)) \leq \lambda_{\max }(\mathbf{M})\|x(k)\|^{2} .
$$

Furthermore, it can be shown that

$$
\begin{aligned}
\lambda_{\min }\left(\operatorname{diag}\left(P,\left(B^{T} P B\right)^{-1}\right)\right) & \|\zeta(k)\|^{2} \leq V(\zeta(k)) \\
& \leq \lambda_{\max }\left(\operatorname{diag}\left(P,\left(B^{T} P B\right)^{-1}\right)\right)\|\zeta(k)\|^{2},
\end{aligned}
$$

Therefore, from (37) and (39) one can derive that

$$
\Delta V(\zeta(k)) \leq-\frac{\hat{\eta}}{\lambda_{\max }(\mathbf{M})} V(\zeta(k))+\gamma
$$


where, due to the continuity assumption mentioned in (14), $\gamma=\tau^{2}\left\|\mho+2 B^{T} P B\right\|\left\|\mathscr{F}^{-}\right\|^{2}$. Note that from (27) it can simply be written that

$$
x^{T}(k) \Omega_{11} x(k)=\left.V(\zeta(k+1))\right|_{f_{\vartheta}(k)=0}-V(\zeta(k))<-\eta x^{T}(k) x(k) .
$$

It is known that $\left.V(\zeta(k+1))\right|_{f_{\vartheta}(k)=0} \geq 0$, and thus, from (42) and (39), it can be claimed that $\lambda_{\max }(\mathbf{M})>\eta$. Therefore

$$
\frac{\hat{\eta}}{\lambda_{\max }(\mathbf{M})}<1
$$

Thus, from Lemma 3, (40) and (41), the bound in (32) can be obtained.

Now let us consider how to solve the inequality (36). By the aid of Lemma 1 and the Schur complement, it can be shown that for the given $P>0$ and $\eta>0$, this inequality can be implied by the LMI in (33).

To be more specific, if one utilizes the controller in (18) $\left(\mathscr{C}_{1}\right), \tau_{1}=2$ in (13) and $\gamma_{1}=4\|\mho+2 S B\|\left\|\mathscr{F}^{-}\right\|^{2}$ in (32). Note that this bound results from the worst case scenario. However, if the sign function $\operatorname{sgn}\left(\hat{f}(k)-\mathscr{F}^{+}\right)$can predict perfectly the location of $f(k)$, which is assumed to be the most cases for slow disturbances, this bound can be reduced to $\tau_{1}^{\star}=1$ and $\gamma_{1}^{\star}=\|\mho \mho+2 S B\|\left\|\mathscr{F}^{-}\right\|^{2}$.

On the other hand, utilizing the controller in $(21)\left(\mathscr{C}_{2}\right)$, we have $\tau_{2}=1.5$ and $\gamma_{2}=$ $2.25\|\mho+2 S B\|\left\|\mathscr{F}^{-}\right\|^{2}$. It should be noted that this bound is also the worst case scenario bound. Since it is assumed that disturbance in the system (1) is slow, this bound, with perfect position estimation, can be reduced to $\tau_{2}^{\star}=0.5$ and $\gamma_{2}^{\star}=$ $0.25\|\mho+2 S B\|\left\|\mathscr{F}^{-}\right\|^{2}$.

\section{Exploiting Disturbance Estimate in the Control Law: $\mathscr{C}_{3}$}

According to the paper [10], for smooth disturbances, $f(k-1)$ is a good approximation to $f(k)$ so as to reduce the ultimate bound on the system state. But, 
unlike [10] in which the system is not uncertain and just involves exogenous disturbance, in this paper we consider a discrete-time system involving uncertainty and exogenous disturbance. Due to the presence of system uncertainty, as seen in (15), we do not have direct access to $f(k-1)$, thus $\hat{f}(k)$ in (16) is used here instead. Furthermore, using the term $\hat{f}(k)$ in the controller directly, rather than using the ones proposed previously seems to have much better performance. Now, by substituting

$$
\vartheta_{3}(k)=\hat{f}(k)
$$

in (12), the following controller is achieved

$$
u_{3}(k)=-(S B)^{-1} S A x(k)-\hat{f}(k) .
$$

Similar idea can also be found in e.g. [10]. Note that, referring to (16), $\vartheta(k)$ in (43) includes system uncertainty, and thus the condition in (13) does not apply. Therefore, the stability of the closed-loop system should be analyzed again. In the following, we consider the stability of the system (1) using the controller (44).

By applying the controller (44) to the system (1), we have

$$
x(k+1)=\left(A+\Delta A_{k}-\hat{A}\right) x(k)-B(S B)^{-1} S \Delta A_{k-1} x(k-1)+B f_{d}(k),
$$

where $f_{d}(k) \triangleq f(k)-f(k-1)$ and $\hat{A} \triangleq B(S B)^{-1} S A$. As seen, the closed-loop system (45) involves time-delay. Furthermore, it can be found that

$$
\sigma_{x}(k+1)=S \Delta A_{k} x(k)-S \Delta A_{k-1} x(k-1)+S B f_{d}(k)
$$

Theorem 3. In the absence of disturbance $f(k)$, the control law (44), (16) can drive the system state onto the ideal sliding surface (6) and the system state is stabilized, if there exist symmetric matrices $\bar{P}>0$ and $\bar{Q}>0$, matrices $X$ and $Y$ 
and also scalars $\varepsilon_{1}>0, \varepsilon_{2}>0$ and $\eta_{1}>0$ satisfying the following LMI:

$$
\left[\begin{array}{cccccccc}
\hat{\mathscr{M}}_{11} & \star & \star & \star & \star & \star & \star & \star \\
0 & -\bar{Q} & \star & \star & \star & \star & \star & \star \\
0 & 0 & \hat{\mathscr{M}}_{22} & \star & \star & \star & \star & \star \\
A \bar{P}+B X & 0 & \sqrt{2} \varepsilon_{1} M M^{T} & -\bar{P}+\varepsilon_{1} M M^{T} & \star & \star & \star & \star \\
B Y & 0 & 0 & 0 & -\bar{P} & \star & \star & \star \\
\bar{P} & 0 & 0 & 0 & 0 & -\bar{\eta}_{1} I & \star & \star \\
N \bar{P} & 0 & 0 & 0 & 0 & 0 & -\varepsilon_{1} I & \star \\
0 & N \bar{P} & 0 & 0 & 0 & 0 & 0 & -\varepsilon_{2} I
\end{array}\right]<0
$$

where $\hat{\mathscr{M}}_{11}=-\bar{P}+\bar{Q}+Y^{T} B^{T}+B Y, \hat{\mathscr{M}}_{22}=-\bar{P}+2\left(\varepsilon_{1}+\varepsilon_{2}\right) M M^{T}$. Here $M$ and $N$ are known matrices in (2), and $S=B^{T} \bar{P}^{-1}$.

ProOF. Refer to Appendix section.

It should be pointed out that the above theorem provides a method to design the disturbance observer based DSMC in order to stabilize the system in (1). However, it does not give a bound on the system states. The following theorem characterizes the boundedness of the obtained closed-loop system state and associated sliding function.

Theorem 4. In the presence of disturbance $f(k)$ satisfying (14), if the LMI in (47) is feasible, for the obtained $P=\bar{P}^{-1}, Q=P \bar{Q} P, \eta_{1}=\bar{\eta}_{1}^{-1}$, the control law (44), (16) will lead to a bound on the augmented system state $\zeta(k)=\left[x^{T}(k), x^{T}(k-\right.$ 1), $\left.\sigma_{x}^{T}(k)\right]^{T}$ as follows:

$$
\begin{aligned}
& \forall v>0, \exists k^{\star}>0, \text { s.t. } \forall k>k^{\star}, \\
& \|\zeta(k)\|^{2} \leq \frac{\lambda_{\max }(\operatorname{diag}(\mathbf{M}, Q))}{\hat{\eta}_{1} \lambda_{\min }\left(\operatorname{diag}\left(P, Q,\left(B^{T} P B\right)^{-1}\right)\right)} \hat{\gamma}+v,
\end{aligned}
$$


where $\mathbf{M}=P B\left(B^{T} P B\right)^{-1} B^{T} P+P$, and $\hat{\gamma}=\left\|\hat{\mho}+2 B^{T} P B\right\| L_{f}^{2} T_{s}^{2}$; here the scalar variable $\hat{\eta}_{1}>0$ and matrix variable $\hat{\mho}>0$ are obtained from solving the following LMI:

$$
\left[\begin{array}{ccccc}
\left(\hat{\eta}_{1}-\eta_{1}\right) I+4 \hat{\varepsilon}_{1} N^{T} N & \star & \star & \star & \star \\
0 & \left(\hat{\eta}_{1}-\eta_{1}\right) I+4 \hat{\varepsilon}_{2} N^{T} N & \star & \star & \star \\
0 & 0 & -\hat{\mho} & \star & \star \\
0 & 0 & M^{T} P B & -\hat{\varepsilon}_{1} I & \star \\
0 & 0 & M^{T} P B & 0 & -\hat{\varepsilon}_{2} I
\end{array}\right]<0,
$$

where $M$ and $N$ are known matrices in (2), $\hat{\varepsilon}_{1}>0$ and $\hat{\varepsilon}_{2}>0$ are scalar variables.

PROOF. The proof of this theorem is an application of the proof of Theorem 2 and thus is omitted here for the brevity purposes.

Remark 5. As seen, applying the controller $\mathscr{C}_{3}$ to the system (1) results in $\hat{\gamma}_{3}=$ $\left\|\hat{\mho}+2 B^{T} P B\right\| L_{f}^{2} T_{s}^{2}$ in (48). Obviously, due to the much smaller $L_{f}^{2} T_{s}^{2}$ in $\hat{\gamma}_{3}$, which is of $O\left(T_{s}^{2}\right)$, the thickness of the boundary layer is reduced, compared to its previous counterparts which are of $O\left(T_{s}\right)$, for the smooth disturbance $f(k)$ satisfying (14).

\section{Simulation Results}

In order to study the performance of the proposed control law, an un-interruptible power system (UPS) is considered here [23]. The aim is to control the PWM inverter in order to keep the output AC voltage at the desired setting robustly. The UPS's capacity is $1 \mathrm{KVA}$. The discrete-time model is obtained with the sampling time $0.01 s$ at the half-load operating point, which is as follows

$$
A=\left[\begin{array}{ccc}
0.9226 & -0.6330 & 0 \\
1.0 & 0 & 0 \\
0 & 1.0 & 0
\end{array}\right], B=\left[\begin{array}{l}
1 \\
0 \\
0
\end{array}\right]
$$


We assume that all the system states are accessible. We also consider the following uncertainty parameters and disturbance in the system

$$
\begin{aligned}
M & =\left[\begin{array}{lll}
0.05 & 0.15 & 0.08
\end{array}\right]^{T}, N=\left[\begin{array}{lll}
-0.05 & 0.06 & 0.10
\end{array}\right], \\
R(k) & =0.3 \sin (k) .
\end{aligned}
$$

Note that the open-loop system is unstable. Notice also that the given DSMC in [15] is not applicable to this system, since the designing LMIs are not feasible. Suppose

$$
d(k)=0.1\left(2-\sin \left(\frac{k}{5}\right)\right) .
$$

Solving the LMI (24) gives the following results:

$$
\begin{aligned}
\bar{P} & =\left[\begin{array}{ccc}
51.5428 & 0.0164 & 0.2975 \\
0.0164 & 54.4440 & 0.3715 \\
0.2975 & 0.3715 & 105.8332
\end{array}\right], \bar{\eta}=175.5974, \varepsilon=96.6945 \\
S & =\left[\begin{array}{lll}
0.0194 & -0.0000 & -0.0001
\end{array}\right]
\end{aligned}
$$

Hence, using $P=\bar{P}^{-1}, \mathscr{F}^{+}=0.2$ and $\mathscr{F}^{-}=0.1$, the control laws $\mathscr{C}_{1}$ and $\mathscr{C}_{2}$ given in (18) and (21), respectively, are obtained. The results by applying these controllers, in addition to the linear controller and the DSMC utilizing only the mean value of the disturbance, to the system (1) are shown in Figs. 2-5. Here, the initial state is assumed to be $x(0)=\left[\begin{array}{lll}1 & 0 & 0\end{array}\right]^{T}$. It can be seen that the system state is bounded and also during the sliding motion the state trajectories are within a boundary layer around the sliding surface $\sigma_{x}(k)=0$.

As seen, for the slow disturbance $f(k)$, in terms of ultimate bound on the system state and also thickness of the boundary layer around the ideal sliding surface, among these four controllers, the controller $\mathscr{C}_{2}$ has the best performance.

As mentioned in Remark 2, in [15] the following control law is proposed: 

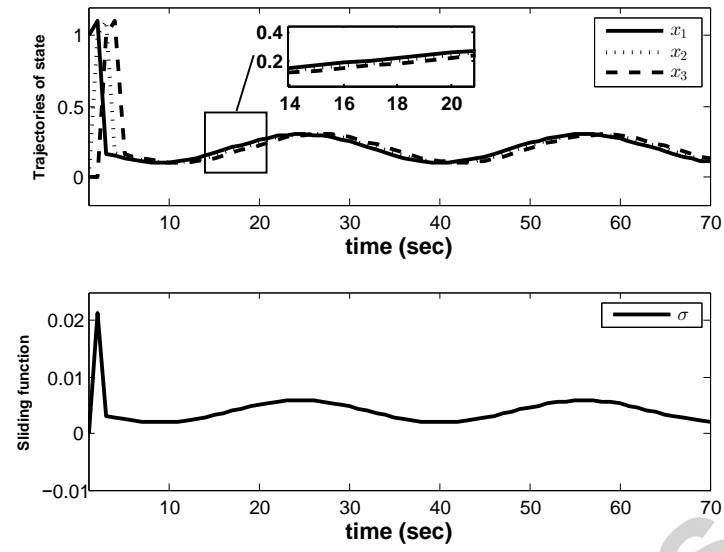

Figure 2: Evolution of the system state and sliding function using linear controller
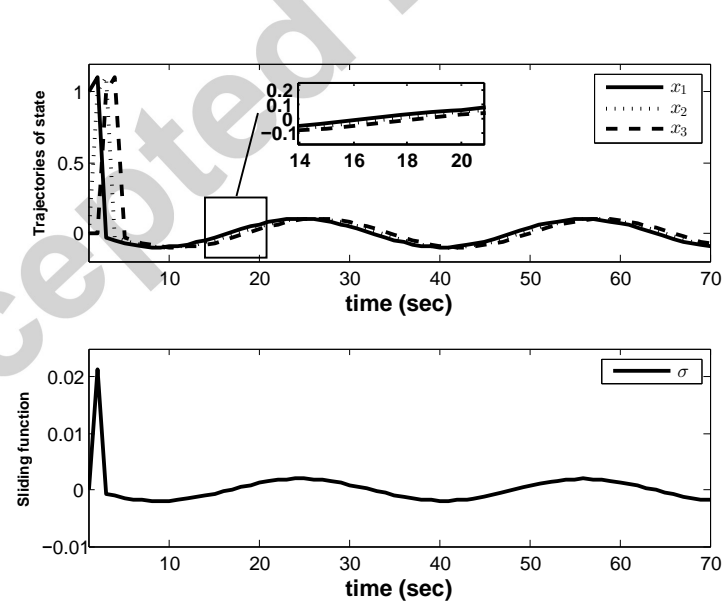

Figure 3: Evolution of the system state and sliding function using mean value of disturbance in DSMC 

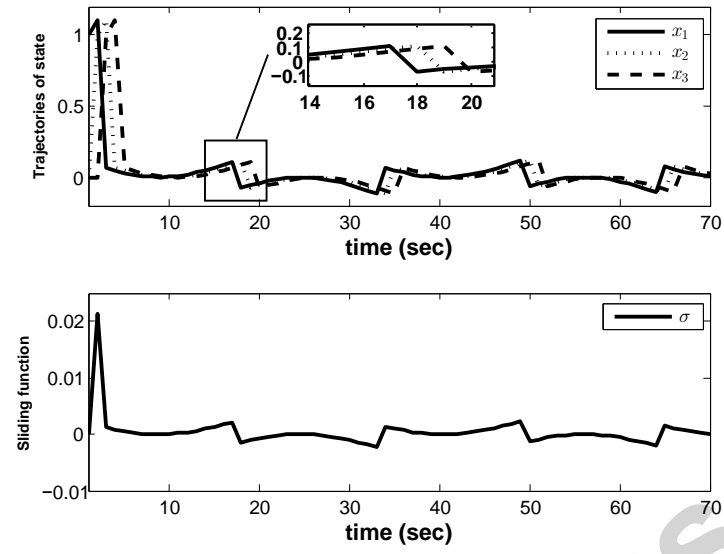

Figure 4: Evolution of the system state and sliding function using $\mathscr{C}_{1}$
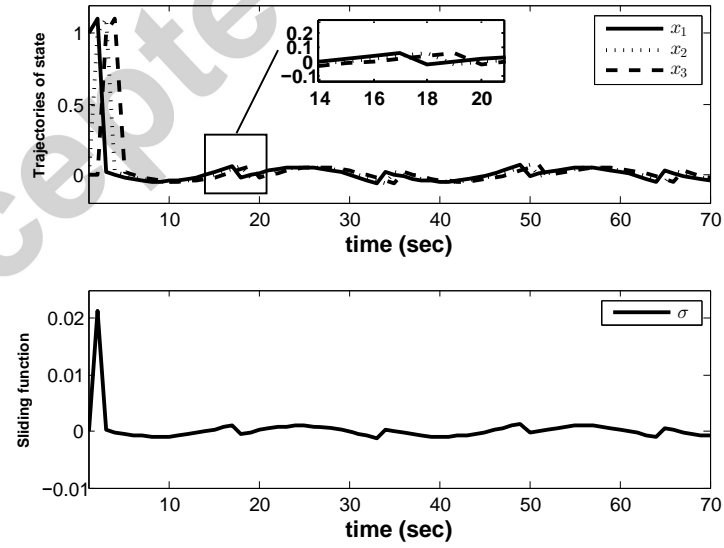

Figure 5: Evolution of the system state and sliding function using $\mathscr{C}_{2}$ 

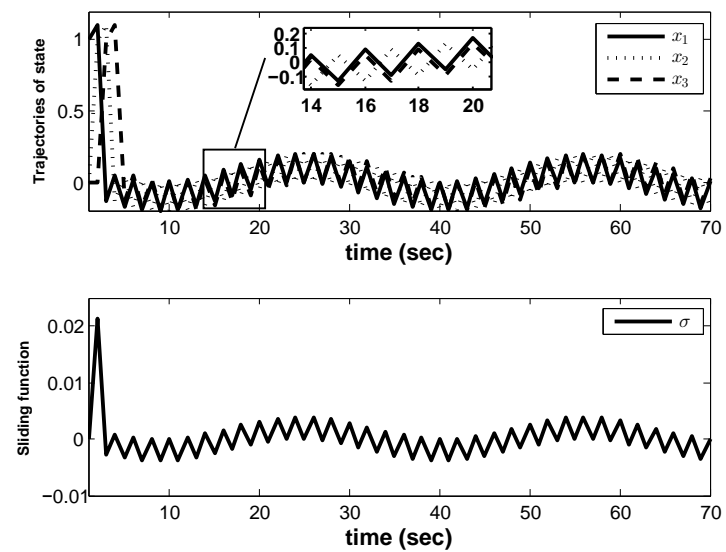

Figure 6: Evolution of the system state and sliding function using the controller in [15]

$$
u(k)=-(S B)^{-1} S A x(k)-\mathscr{F}^{+}-\operatorname{diag}\left(\mathscr{F}^{-}\right) \operatorname{sgn}\left(\sigma_{x}(k)\right)
$$

Figure 6 shows the results by applying this controller to the system (1). Note that as the LMI condition in [15] is not feasible, the controller (50) is constructed by the choice of $S$ achieved through solving the LMI in (24). This indeed shows the superiority of our approaches compared to the existing literature. As it is emphasized in [5], using this controller leads state trajectories to chatter around the switching surface with amplitude dependent on the lower bound of the component in (19) and with the frequency equal to the sampling rate. As it was mentioned in Remark 2, using the controllers $\mathscr{C}_{1}$ and $\mathscr{C}_{2}$, while the chattering still happens, in this case, the state trajectories chatter with the frequency equal to the frequency of exogenous disturbance.

Now, solving the LMI in (47), the following results are obtained: 

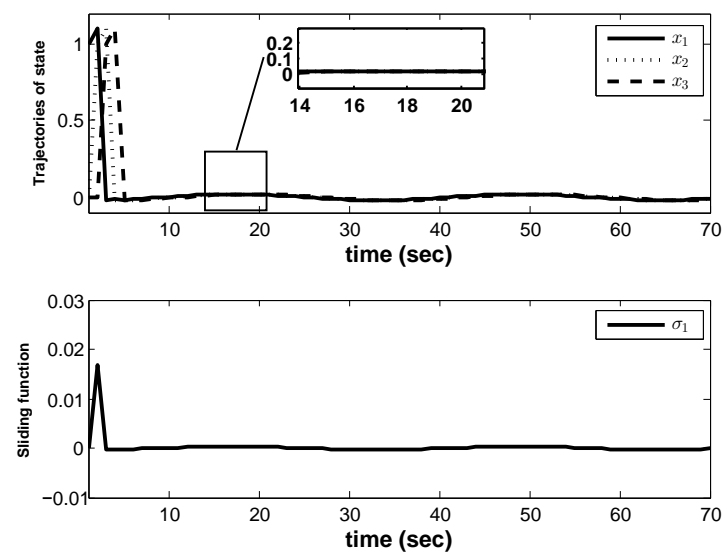

Figure 7: Evolution of the system state and sliding function using $\mathscr{C}_{3}$

$$
\begin{gathered}
\bar{P}=\left[\begin{array}{ccc}
65.3131 & -0.2883 & 0.5724 \\
-0.2883 & 71.5406 & 0.1175 \\
0.5724 & 0.1175 & 152.0610
\end{array}\right], \bar{Q}=\left[\begin{array}{ccc}
38.6665 & -0.3757 & 0.0127 \\
-0.3757 & 19.9712 & -0.0472 \\
0.0127 & -0.0472 & 76.8444
\end{array}\right] \\
\bar{\eta}_{1}=414.0327, \varepsilon_{1}=150.6769, \varepsilon_{2}=171.9258 \\
S=\left[\begin{array}{lll}
0.0153 & 0.0001 & -0.0001
\end{array}\right]
\end{gathered}
$$

Figure 7 shows the results of applying the control law $\mathscr{C}_{3}$ in (44) to the system (1). As mentioned, this controller uses disturbance estimate. It is crystal clear that this controller has the best performance compared to the previous controllers, in terms of ultimate bound on system state, for the systems involving smooth disturbances. Notice that as the choice of sliding matrix in the last controller $\mathscr{C}_{3}$ is not the same as the one used in the previous controllers, it is hard to compare the current results in terms of the bounds on the systems states and, in addition, the thickness of the obtained boundary layer around the ideal sliding function. To have a fair 
comparison, we need to use the same $S$ in all the controllers. It is also not hard to show that if the LMI in (47) is feasible then the LMI in (24) will necessarily be feasible, and hence, we can use the the sliding matrix obtained from the LMI in (47) to construct the aforementioned controllers. Table 1 illustrates the obtained results. As seen in Table $1, \mathscr{C}_{3}$, with the assumption of having a system involving

Table 1: Comparison of the controllers

\begin{tabular}{ccc}
\hline \hline Controller & bound on the system state $(\|x(k)\|)$ & boundary layer thickness $\left(\left\|\sigma_{x}(k)\right\|\right)$ \\
\hline Linear controller & 0.5152 & 0.0046 \\
Controller utilizing the mean value of disturbance & 0.1719 & 0.0019 \\
$\mathscr{C}_{1}$ & 0.1709 & 0.0018 \\
$\mathscr{C}_{2}$ & 0.0868 & 0.0010 \\
Controller in (50) & 0.2821 & 0.0031 \\
$\mathscr{C}_{3}$ & 0.0342 & 0.00003 \\
\hline
\end{tabular}

slow disturbances, perfectly outperforms the other two controllers, however, as it was mentioned earlier, at the expense of dealing with higher order systems and implementing more intensive computations. Moreover, it can be seen from Figure 4 and 5 that $\mathscr{C}_{2}$ has resulted in slightly smoother state trajectories. Clearly, the performances of the controllers $\mathscr{C}_{1}, \mathscr{C}_{2}, \mathscr{C}_{3}$ and even the controller utilizing the mean value of disturbance outperform that of the controller in (50), and the rate of chattering occurred with (50) is much higher.

\section{Conclusions}

In this paper, a new LMI based robust DSMC for the systems involving unmatched uncertainty and matched disturbance has been developed. The proposed LMI method is applicable to general systems including unstable systems. Furthermore, some notes on the use of the discontinuous term in the discrete-time 
sliding mode controller have been given and two new switching function has been developed. Inspired by the idea of disturbance observer, a new controller for the underlying uncertain systems has been proposed in this paper. The controller with disturbance estimator outperforms the other kind of DSMCs, including linear controller and DSMCs using discontinuous components, while the underlying systems involves slow exogenous disturbances. Nevertheless, the downside is that, since the order of the closed-loop system increases, the scheme using DSMC with disturbance estimator is more computationally intensive.

\section{Appendix A. Proof of Theorem 3}

Define

$$
V(\zeta(k))=x^{T}(k) P x(k)+x^{T}(k-1) Q x(k-1)+\sigma_{x}^{T}(k)(S B)^{-1} \sigma_{x}(k),
$$

where $\zeta(k)=\left[\begin{array}{lll}x^{T}(k) & x^{T}(k-1) & \sigma_{x}^{T}(k)\end{array}\right]^{T}, P>0$ and $Q>0$ are symmetric matrices and $S=B^{T} P$. Thus, we can write

$$
\begin{aligned}
\Delta V(\zeta(k))= & V(\zeta(k+1))-V(\zeta(k)) \\
= & x^{T}(k+1) P x(k+1)+x^{T}(k) Q x(k)+\sigma_{x}^{T}(k+1)(S B)^{-1} \sigma_{x}(k+1)-x^{T}(k) P x(k) \\
& -x^{T}(k-1) Q x(k-1)-\sigma_{x}^{T}(k)(S B)^{-1} \sigma_{x}(k) .
\end{aligned}
$$

Now, it can be shown that

$$
\Delta V(\zeta(k))=\left[\begin{array}{c}
x(k) \\
x(k-1) \\
f_{d}(k)
\end{array}\right]^{T}\left[\begin{array}{ccc}
\Sigma_{11} & \Sigma_{12} & \Sigma_{13} \\
\Sigma_{12}^{T} & \Sigma_{22} & \Sigma_{23} \\
\Sigma_{13}^{T} & \Sigma_{23}^{T} & \Sigma_{33}
\end{array}\right]\left[\begin{array}{c}
x(k) \\
x(k-1) \\
f_{d}(k)
\end{array}\right]
$$


where

$$
\begin{aligned}
\Sigma_{11}:= & \left(A+\Delta A_{k}\right)^{T} P\left(A+\Delta A_{k}\right)-\left(A+\Delta A_{k}\right)^{T} P B\left(B^{T} P B\right)^{-1} B^{T} P\left(A+\Delta A_{k}\right) \\
& -P+Q-P B\left(B^{T} P B\right)^{-1} B^{T} P+2 \Delta A_{k}^{T} P B\left(B^{T} P B\right)^{-1} B^{T} P \Delta A_{k}, \\
\Sigma_{12}:= & -2 \Delta A_{k}^{T} S^{T}(S B)^{-1} S \Delta A_{k-1}, \\
\Sigma_{22}:= & 2 \Delta A_{k-1}^{T} P B\left(B^{T} P B\right)^{-1} B^{T} P \Delta A_{k-1}-Q,
\end{aligned}
$$

and $\Sigma_{13}=2 \Delta A_{k}^{T} S^{T}, \Sigma_{23}=-2 \Delta A_{k-1}^{T} S^{T}$ and $\Sigma_{33}=2 S B$. In the absence of the disturbance $f(k), f_{d}(k)=0$. Then the system is stabilized if

$$
\Upsilon:=\left[\begin{array}{ll}
\Sigma_{11} & \Sigma_{12} \\
\Sigma_{12}^{T} & \Sigma_{22}
\end{array}\right]<-\eta_{1} I
$$

where $\eta_{1}>0$ is a scalar variable. Following a similar approach given in the proof of Theorem 1, and by using the Schur complement, Corollary 1, Lemma 2 and Lemma 4, it can be demonstrated that the inequality in (A.3) can be implied by the LMI in (47), where $\bar{Q}=\bar{P} Q \bar{P}, X=F \bar{P}, Y=L \bar{P}$ ( $F$ and $L$ are two auxiliary variables), and $\bar{\eta}_{1}=\eta_{1}^{-1}$.

[1] N. Lai, C. Edwards, S. K. Spurgeon, Discrete output feedback sliding-mode control with integral action, Int. J. Robust Nonlinear Control 16 (2006) 2143.

[2] W. Gao, Y. Wang, A. Homaifa, Discrete-time variable structure control system, IEEE Trans. Ind. Electron 42 (1995) 117-122.

[3] A. Koshkouei, A. Zinober, Sliding mode control of discrete-time systems, Journal of Dynamic Systems, Measurement, and Control 122 (2000) 793802. 
[4] S. Sapturk, Y. Istefanopulous, O. Kaynak, On the stability of discrete-time sliding mode control systems, IEEE Transactions on Automatic Control 32 (1987) 930-932.

[5] S. Hui, S. H. Zak, On discrete-time variable structure sliding mode control, Systems and Control Letters 38 (1999) 283-288.

[6] S. Spurgeon, Hyperplane design techniques for discrete-time variable structure control systems, International Journal of Control 55(2) (1992) 445-456.

[7] G. Monsees, Discrete-time sliding mode control, Ph.D. Dissertation, Delft University of Technolog, The Netherlands, 2002.

[8] J. Manela, Deterministic control of uncertain linear discrete and sampleddata systems, Ph.D. Dissertation, University of California, Berkeley, 1985.

[9] M. Corless, Stabilization of uncertain discrete-time systems, in: Proceedings of the IFAC Workshop on Model Error Concepts and Compensation, Boston, 1985, pp. 125-128.

[10] W. Su, S. Drakunov, Ü. Özgüner, An $\mathrm{O}\left(\mathrm{T}^{2}\right)$ boundary layer in sliding mode for sampled-data systems, IEEE Transactions on Automatic Control 45 (2000) 482-485.

[11] M. Lješnjanin, B. Draženović, Č. Milosavljević, B. Veselić, Disturbance compensation in digital sliding mode, in: International Conference on Computer as a Tool (EUROCON), IEEE, 2011, pp. 1-4.

[12] C. Milosavljevic, B. Perunicic-Drazenovic, B. Veselic, Discrete-time velocity servo system design using sliding mode control approach with dis- 
turbance compensation, IEEE Transactions on Industrial Informatics 9 (2) (2013) 920-927.

[13] V. Acary, B. Brogliato, Implicit Euler numerical scheme and chattering-free implementation of sliding mode systems, Systems \& Control Letters 59 (5) (2010) 284-293.

[14] O. Huber, V. Acary, B. Brogliato, F. Plestan, Discrete-time twisting controller without numerical chattering: analysis and experimental results with an implicit method, in: 53rd Annual Conference on Decision and Control (CDC), IEEE, 2014, pp. 4373-4378.

[15] Y. Niu, D. Ho, Design of sliding mode control subject to packet losses, IEEE Transactions on Automatic Control 55 (2010) 2623-2628.

[16] T. Jiaa, Y. Niu, Y. Zoua, Sliding mode control for stochastic systems subject to packet losses, Information Sciences 217 (2012) 117-126.

[17] Y. Niu, D. Ho, J. Lam, Robust integral sliding mode control for uncertain stochastic systems with time-varying delay, Automatica 41 (2005) 873-880.

[18] I. Peterson, A stabilization algorithm for a class of uncertain linear systems, Systems Control Lett. 8 (1987) 351-357.

[19] L. Li, V. Ugrinovskii, R. Orsi, Decentralized robust control of uncertain Markov jump parameter systems via output feedback, Automatica 43 (2007) 1932-1944.

[20] A. Bartoszewicz, Discrete-time quasi-sliding-mode control strategies, IEEE Transactions on Industrial Electronics 45 (1998) 633-637. 
[21] S. Govindaswamy, S. Spurgeon, T. Floquet, Discrete-time output feedback sliding-mode control design for uncertain systems using linear matrix inequalities, International Journal of Control 84 (2011) 916-930.

[22] H. Khalil, Nonlinear Systems, 3rd Edition, Prentice Hall, New York, 2002.

[23] F. W. Yang, Z. D. Wang, Y. S. Hung, M. Gani, $\mathrm{H}_{\infty}$ control for networked systems with random communication delays, IEEE Trans. Autom. Control 51 (3) (2006) 511-518. 